\title{
The Role of The Medial Buttress Plate In The Treatment of Pauwels Type $\square$ And $\square$ Femoral Neck Fracture: A Retrospective Study And Preliminary Results
}

\section{Chao Ma}

The First Affiliated Hospital of Xinjiang Medical University

Yanshi Liu

The First Affiliated Hospital of Xinjiang Medical University

Jialin Liu

The First Affiliated Hospital of Xinjiang Medical University

\section{Li Chen}

The First Affiliated Hospital of Xinjiang Medical University

Jinyong Huang

The First Affiliated Hospital of Xinjiang Medical University

\section{Xuefeng Luo}

The First Affiliated Hospital of Xinjiang Medical University

\section{Zengru Xie (D3034@xjmu.edu.cn )}

The First Affiliated Hospital of Xinjiang Medical University

\section{Research Article}

Keywords: Buttress plate, Multiple cannulated screws, Femoral neck fracture, Pauwels classification

Posted Date: June 21st, 2021

DOl: https://doi.org/10.21203/rs.3.rs-571550/v1

License: (a) (i) This work is licensed under a Creative Commons Attribution 4.0 International License. Read Full License 


\section{Abstract}

Background: The purpose of this study was to compare the effectiveness of multiple cannulated screws combined with medial buttress plate or not for the treatment of unstable femoral neck fracture.

Methods: 69 nonelderly patients with Garden type III-IV femoral neck fracture were retrospectively analyzed. The patients were divided into MCS (multiple cannulated screws) group and CMBP (combined with medial buttress plate) group according to the surgical method. Patient's demographic data, Harris Hip Score, EQ-5D index and complications at minimum of 2 years follow-up were analyzed.

Results: There were 47 patients in the MCS group (35 male and 12 females) with a mean age of $40.28 \pm 12.64$ years, whereas 22 patients in the CMBP group (17 male and 5 females) with a mean age of $43.86 \pm 12.55$ years. In the MCS group, there were one avascular necrosis, five postoperative nonunion, five implant failure, and two femoral neck shortening. While one implant failure, two postoperative nonunion and two impingement in the CMBP group. The difference of HHS scores was statistically significant at 3 months after surgery between the two groups $(P<0.05)$, whereas there was no statistical significance at 6 months, 1 year and 2 years $(P>0.05)$. The same results were found in EQ-5D index.

Conclusions: Anatomic reduction and stable fixation contribute to satisfactory outcomes in the treatment of nonelderly displaced femoral neck fractures. A buttress plate in the medial region of the femur neck with cannulated screws offers patients the best biomechanical microenvironment needed for fracture

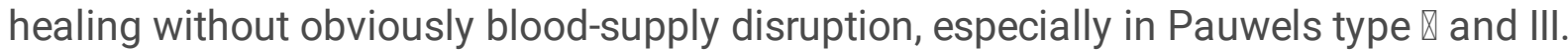

\section{Background}

Femoral neck fractures in healthy nonelderly individuals are relatively uncommon and are usually caused by high energy trauma ${ }^{1}$. Prompt diagnosis, anatomic reduction and stable internal fixation may contribute the satisfactory outcomes ${ }^{1-3}$. However, the optimal definitive management of such fractures remains controversial and a challenging orthopaedic problem due to the complexity of anatomical relationship and a vulnerable blood supply to the femoral head, which could result in malunion, nonunion, or avascular necrosis ${ }^{4-6}$.

The orthopaedic surgeon's goals of obtaining and maintaining anatomic reduction until bony union have been addressed by a number of surgical approaches and fixation constructs, including a sliding hip screw device, proximal femoral locking plates, cephalomedullary nails, and multiple cannulated parallel lag screws $^{7-9}$. However, even with these treatment methods, complications are still common. Additionally, for all these injuries, there is no consensus on which single fixation option is superior ${ }^{7}$. Mir et al. ${ }^{3}$ proposed that anatomic reduction and fixation, coupled with a medial buttress plate that resists shearing forces, may reduce the historically high complication rate of vertical femoral neck fractures. This technique has not been widely applied to this injury pattern, and there are little published data demonstrating the 
outcomes. The purpose of this study was to compare the effectiveness of multiple cannulated screws combined with medial buttress plate or not for the treatment of unstable femoral neck fracture.

\section{Methods}

The retrospective study included 342 patients who underwent femoral neck fractures treated at our institution from April 2012 to February 2018.

The inclusion criteria were: (1) age between 18 to 65 years, (2) femoral neck fracture, Garden type III-IV, (3) fixation should be conducted less than 10 days from injury, (4) American Academy of Anesthesiology class 1 or 2, (5) no other fracture in the lower limb, (6) a minimum of 2 years follow-up. The exclusion criteria were (1) pathological fractures, (2) severe blood and immune system diseases, (3) severe multiple traumas or a previous history of ipsilateral hip or femur surgery, and (4) conditions such as osteoarthritis and post-dysplastic deformities. A total of 69 eligible patients were reviewed. There were 52 males and 17 females, with a mean age of $41.42 \pm 12.63$ years. Of all the patients, 47 patients were in MCS (multiple cannulated screws) group and 22 patients were in CMBP (combined with medial buttress plate) group.

\section{Surgical procedures}

Patients in both groups were under general anesthesia, lying supine on the operating table with the affected hip elevated at an angle of $10-15^{\circ}$. A C-arm x-ray machine was used to take anteroposterior and lateral radiographs of the femoral neck. The reduction was considered acceptable if the angle between the medial femoral head and the medial femoral shaft cortex in the anteroposterior and lateral radiographs met the Garden Alignment Index Grade I criteria. Otherwise, the effectiveness of the reduction was considered unsatisfactory ${ }^{10}$.

For patients in MCS group, fixations were monitored under C-arm fluoroscopy and conducted with cannulated screws. Three cannulated screws were inserted parallel to each other. Open reduction was considered when anatomic reduction could not be achieved in the patient.

For patients in CMBP group, the modified Smith-Petersen approach was used for this surgery. Anatomic reduction was achieved by a combination of manual traction and femoral head manipulation. In addition, the surgeon can reduce the femoral neck fracture under direct vision. After fluoroscopy confirmed the reduction, a small incision was made on the lateral side of the femur, through which the cannulated screw guide wires were drilled into the femoral head, and three cannulated screws were inserted parallel into the femoral head. The reconstruction plate was then shaped and placed on the anteromedial surface of the femoral neck and fixed with three screws.

\section{Postoperative management}

Patients in both groups received perioperative antibiotics, as well as low molecular weight heparin (4000AxalU) once daily for two weeks as antithrombotic prophylaxis. All patients began postoperative 
quadriceps muscle contraction and relaxation exercises on day 2, CPM (continuous passive motion) training on day 3. Regular follow-ups were performed at 1 months, 3 months, 6 months, 1 year and 2 years after surgery, and instructions for mobilization, including weight-bearing as tolerated were provided based on the results of radiological evaluation. The degree of union, position of implant, and loss of fracture alignment were observed and femoral neck shortening was measured on digital radiographs. The Harris Hip Score $(\mathrm{HHS})^{11}$ was calculated to evaluate hip function, and EQ-5D index (EuroQol fivedimension questionnaire) was used to measure the health-related quality of life. Nonunion was defined as clearly visible fracture line one year postoperatively ${ }^{12}$. Avascular necrosis was identified by the Steinberg classification from stage 2 and upward $^{13}$.

All 69 patients' demographic data, Harris Hip Score, EQ-5D index and complications at minimum of 2 years follow-up were analyzed.

\section{Statistical analysis}

Statistical analysis was performed using SPSS version 22.0 (SPSS Inc., Chicago, IL, USA). All quantitative data are presented as mean \pm standard deviation (SD). The $t$ test for two independent samples was used to analyze the data. Categorical data were compared using the $\chi 2$ test or the Fisher exact probability method if the theoretical frequency was less than 1 . A P value $<0.05$ was considered statistically significant.

\section{Results}

There were 47 patients in the MCS group (35 male and 12 females) with a mean age of $40.28 \pm 12.64$ years, whereas 22 patients in the CMBP group (17 male and 5 females) with a mean age of $43.86 \pm 12.55$ years. The etiology of femoral neck fracture includes traffic accident in 47 cases (33 in MCS and 14 in CMBP), fall in 15 cases ( 9 in MCS and 6 in CMBP), sport injury in 7 cases ( 5 in MCS and 2 in CMBP). According to Garden classification, there were 42 patients in Type $\otimes$ (28 in MCS and 14 in CMBP) and 27 patients in Type $\triangle(19$ in MCS and 8 in CMBP). Based on Pauwels classification, the MCS group includes 11 cases in Type $\nabla, 29$ in Type $\nabla$ and 7 in Type $\varangle$, whilst CMBP group includes 8 cases in Type $\nabla$ and 14 in Type $₫$. With ASA classification, MCS group consists of 32 cases in Grade 1 and 15 in Grade 2, whereas CMBP group contains 16 cases in Grade 1 and 6 in Grade 2. There were no statistically significant differences between the two groups $(P>0.05)$ except for Pauwels classification $(P<0.05)$. (Table 1$)$ 
Table 1

baseline characteristics of patients. Comparison between groups (mean \pm standard deviation).

\begin{tabular}{|c|c|c|c|c|}
\hline & MCS & CMBP & $x^{2} / t$ & P-value \\
\hline Gender & & & 0.063 & 0.801 \\
\hline Male & 35 & 17 & & \\
\hline Female & 12 & 5 & & \\
\hline Age (years) & $40.28 \pm 12.64$ & $43.86 \pm 12.55$ & -1.101 & 0.275 \\
\hline BMI & $24.31 \pm 2.90$ & $24.53 \pm 2.06$ & -0.319 & 0.751 \\
\hline Fractured limb & & & 0.665 & 0.415 \\
\hline Left & 22 & 8 & & \\
\hline Right & 25 & 14 & & \\
\hline Mechanism of injuries & & & 0.585 & 0.746 \\
\hline Traffic accident & 33 & 14 & & \\
\hline Fall & 9 & 6 & & \\
\hline Sport injury & 5 & 2 & & \\
\hline Garden classification & & & 0.104 & 0.747 \\
\hline Type $\otimes$ & 28 & 14 & & \\
\hline Type $\otimes$ & 19 & 8 & & \\
\hline Pauwels classification & & & 19.127 & $P<0.001$ \\
\hline Type $\otimes$ & 11 & 0 & & \\
\hline 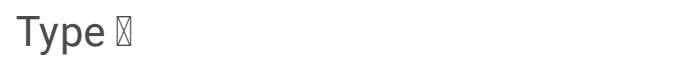 & 29 & 8 & & \\
\hline Type $\otimes$ & 7 & 14 & & \\
\hline ASA classification & & & 0.153 & 0.696 \\
\hline Grade 1 & 32 & 16 & & \\
\hline Grade 2 & 15 & 6 & & \\
\hline Interval from injury to operation (d) & $4.8 \pm 1.6$ & $4.9 \pm 1.8$ & -0.176 & 0.861 \\
\hline Operative time $(\mathrm{min})$ & $52.17 \pm 11.57$ & $92.41 \pm 17.87$ & -11.239 & $P<0.001$ \\
\hline Intraoperative blood loss (ml) & $51.81 \pm 19.21$ & $265.23 \pm 53.15$ & -24.408 & $P<0.001$ \\
\hline
\end{tabular}




\begin{tabular}{|llll|}
\hline \multicolumn{1}{|c|}{ MCS } & CMBP & $\mathrm{X}^{2} / \mathrm{t}$ & P-value \\
\hline MCS: multiple cannulated screws group & & \\
CMBP: combined with medial buttress plate group & & \\
BMI: body mass index & & \\
ASA: American Society of Anesthesiologists & & \\
\hline
\end{tabular}

The mean elapsed time from injury to operation was 4.8 days (range 3 to 8 days) in MCS group, while 4.9 days (range 3 to 9 days) in CMBP group, the difference was not statistically significant $(P>0.05)$. The average operative time was 52.2 minutes (range 29 to 75 minutes) in MCS group and 92.4 minutes (range 74 to 134 minutes) in CMBP group. In addition, the mean intraoperative blood loss was $51.8 \mathrm{ml}$ (range 20 to $100 \mathrm{ml}$ ) in MCS group and $265.2 \mathrm{ml}$ (range 170 to $350 \mathrm{ml}$ ) in CMBP group, respectively. The differences of operative time and intraoperative blood loss between two groups were statistically significant $(P<0.05)$. (Table 1)

All patients in two groups were closely followed up at minimum of two years after the operation. For MCS group, the mean Harris Hip Scores of healed patients were $73.66 \pm 4.29,85.15 \pm 3.92,90.43 \pm 2.38$, and $92.51 \pm 2.80$ at 3 months, 6 months, 1 year and 2 years after surgery respectively, while $76.18 \pm 4.12$, $88.04 \pm 7.43,91.23 \pm 3.66$, and $92.91 \pm 2.93$ in CMBP group. As for EQ-5D index, the average scores were $0.61 \pm 0.16,0.68 \pm 0.14,0.77 \pm 0.11$, and $0.81 \pm 0.12$ at 3 months, 6 months, 1 year and 2 years after surgery respectively in MCS group. The average scores were $0.70 \pm 0.12,0.74 \pm 0.11,0.79 \pm 0.17$, and 0.82 \pm 0.14 at 3 months, 6 months, 1 year and 2 years after surgery respectively in CMBP group. There were significant differences between the two groups at 3 months and 6 months after surgery $(P<0.05)$, while no obvious difference at 1 year and 2 years $(P>0,05)$. (Table 2$)$ 
Table 2

Functional outcome by HHS score and EQ-5D index. Comparison between groups (mean \pm standard deviation).

\begin{tabular}{|lllll|}
\hline Functional outcome & MCS & CMBP & t & P-value \\
\hline HHS score & & & & \\
\hline 3 months & $73.66 \pm 4.30$ & $76.18 \pm 4.13$ & -2.302 & 0.024 \\
\hline 6 months & $85.15 \pm 3.92$ & $88.05 \pm 7.44$ & -2.123 & 0.037 \\
\hline 12 months & $90.43 \pm 2.38$ & $91.23 \pm 3.66$ & -1.092 & 0.279 \\
\hline 24 months & $92.51 \pm 2.80$ & $92.91 \pm 2.93$ & -0.543 & 0.589 \\
\hline EQ-5D index & & & & \\
\hline 3 months & $0.61 \pm 0.16$ & $0.70 \pm 0.12$ & -2.385 & 0.020 \\
\hline 6 months & $0.68 \pm 0.14$ & $0.74 \pm 0.11$ & -2.022 & 0.047 \\
\hline 12 months & $0.77 \pm 0.11$ & $0.79 \pm 0.17$ & -0.792 & 0.431 \\
\hline 24 months & $0.81 \pm 0.12$ & $0.82 \pm 0.14$ & -0.372 & 0.711 \\
\hline MCS: multiple cannulated screws group & & \\
CMBP: combined with medial buttress plate group & & \\
HHS: Harris Hip Score & & & \\
EQ-5D: EuroQol five dimensions questionnaire & & \\
\hline
\end{tabular}

The differences between MCS group and CMBP group in the treatment of unstable femoral neck fracture (Pauwels classification, Type $\Downarrow$ and Type $\Downarrow$ ) were shown in Fig. 1. The difference of HHS scores was statistically significant at 3 months after surgery $(P<0.05)$, whereas there was no statistical significance at 6 months, 1 year and 2 years $(P>0.05)$. The same results were found in EQ-5D index.

The incidence of complications related to the fracture are shown in Table 3. During the follow-up period in MCS group, one patient developed to avascular necrosis. Postoperative nonunion occurred in 5 patients, three of these patients developed cannulated screw back out. Femoral neck shortening occurred in two cases in which cannulated screw back out and screw broken occurred in one case respectively, but the fracture appeared radiographically healed in a malunited position. Six patients underwent hip arthroplasty in the second phase. 
Table 3

Incidence of complications related to the fracture

\begin{tabular}{|lll|}
\hline Complications & MCS (n/N) & CMBP (n/N) \\
\hline Avascular necrosis & $1(2.1 \%)$ & $0(0 \%)$ \\
\hline Superficial or wound infection & $0(0 \%)$ & $0(0 \%)$ \\
\hline $\begin{array}{l}\text { Implant failure (cannulated screw back out/screw } \\
\text { or plate breakage) }\end{array}$ & $5(10.6 \%)$ & $1(4.5 \%)$ \\
\hline Nonunion & $5(10.6 \%)$ & $2(9.1 \%)$ \\
\hline Impingement & $0(0 \%)$ & $2(9.1 \%)$ \\
\hline Femoral neck shortening & $2(4.3 \%)$ & $0(0 \%)$ \\
\hline MCS: multiple cannulated screws group & & \\
CMBP: combined with medial buttress plate group & & \\
\hline
\end{tabular}

In the CMBP group, nonunion observed in 2 cases, of which one developed cannulated screw back out, and they were all managed by hip arthroplasty. Two patients felt groin pain with deep hip joint flexion, because of impingement between the high position plate and acetabulum or labrum when the hip is in deep flexion, but one resolved by 3 months post-operatively and the other one received removal of the medial buttress plate. There was no superficial or wound infection observed in both groups.

\section{Discussion}

Compressive stress, tensile stress and shear force are generated at the proximal segment of the femur during force conduction, because of the femoral neck shaft angle. Stress trabecula bone, tension trabecula bone and calcar femorale are derived based on the loading and offered the ability of mechanotransduction. When femoral neck fracture occurred, the normal stress conduction is lost because of these destroyed truss structures. Therefore, it is generally accepted that anatomic reduction with stable fixation offers patients the best biomechanical microenvironment needed for fracture healing without complication to maintain the native hip ${ }^{14,15}$.

The mechanical stability of fracture fixation structure is closely related to the implant, reduction quality as well as the structure and material characteristics of bone ${ }^{9}$. Kunapuli et al. ${ }^{16}$ found that the medial femoral neck buttress plate enhanced the stability of cannulated screw and sliding hip screw constructs, significantly increased stiffness and load to failure. Giorfano et al. ${ }^{17}$ conducted a mechanical study to analyze the role of the medial buttress plate in Pauwels type III femoral neck fractures by comparing the resistance of two fixation methods using three cannulated screws, and the results showed that the medial buttress plate combined with multiple cannulated screw fixation provided a mechanically superior structure for Pauwels type III fractures. Additionally, Nwankwo et al. ${ }^{15}$ conducted a mechanical test with 
ten matched pairs of young cadaveric femurs and concluded that the application of medial plates to Pauwels Type III femoral neck fractures significantly decreased angular displacement and shear.

In the present study, two patients (4.3\%) suffered femoral neck shortening caused by implant failure in MCS group, while none was observed in CMBP group. Three cases $(6.4 \%)$ developed postoperative nonunion with cannulated screw back out in MCS group and one (4.5\%) in CMBP group. Nonunion is the main complication following internal fixation of the femoral neck fractures ${ }^{18}$. The $91 \%$ union rate in CMBP group is favorable comparable to the study for Pauwels type III fractures, in which union rates ranging from $73.7-89 \% 5,12,19,20$.

We speculate that the satisfactory outcomes probably derived from anatomic reduction under the direct vision and the stable fixation under the combination of intramedullary and extramedullary in the CMBP group. Thus, there is a potential benefit that combine a medial buttress plate with cannulated screws in the anteromedial region of the femur neck improves the mechanical resistance of fixation in unstable fractures, especially in Pauwels type $₫$ and type III.

Preservation of the blood supply to the femoral head is most important in the treatment of femoral neck fractures. The medial circumflex artery (MFCA) is the main vascular supply to the femoral head, and the superior retinacular artery (SRA) is the most important branch ${ }^{21-23}$. The SRA and its branches are closely located to the femoral neck and are easily destroyed in displaced femoral neck fractures ${ }^{22,24}$. It has also been shown that the inferior retinacular artery (IRA) also plays an important role in femoral head perfusion ${ }^{21,25}$. IRA was present in all hips and the point of entry into the hip capsule as well as intraarticular course were consistent. The study by Sara et al. ${ }^{23}$ determined the anatomical (especially in vascular) feasibility of this approach to the treatment of femoral neck fractures via an anterior approach to the hip. They described the location of the vessels around the femoral neck by using a clock-face system, with the IRA coursing posteromedially between 7:00 and 8:00, placing a straight plate medially or slightly anteriorly will not disrupt the IRA. In addition, lesser trochanter can be used as a positioning reference and the safe zone for placing the straight plate is just anterior to the lesser trochanter (5:006:00) when IRA is less well-visualized. The results of these studies not only provided an anatomical basis for the application of the medial femoral neck plate, but will also change the misconception that the plate would disrupts the blood supply to the femoral head.

In the present study, for patients in the CMBP group, the reconstruction plate was placed anteromedially at the femoral neck via a modified Smith-Petersen approach, which is consistent with the recommendations of Sara et.al ${ }^{23}$. Compared with the MCS group, open reduction allowed emptying of the intra-articular hematoma, reducing the risk of avascular necrosis of the femoral head due to high intraarticular pressure ${ }^{26}$. In addition, the femur is usually slightly externally rotated during the procedure to aid in visualization of the femoral neck. At this time, the Weibrecht's ligament and the IRA that travels within are naturally fall away from the femoral neck, reducing the risk of damaging the IRA during the placement of the plate. During the follow-up, the absence of avascular necrosis in the CMBP group supports that this surgical approach does not increase the osteonecrosis rate of Garden type III-IV fractures. On the other 
hand, postoperative nonunion is probably related to blood supply, 2 (4.3\%) patients in the MCS group and $1(4.5 \%)$ in the CMBP group experiencing nonunion excluding implant failure. This supports that the medial buttress plate does not increase the nonunion rate.

As shown in Table 1, there is no doubt that the CMBP group has longer operation time and more intraoperative blood loss. This is acceptable given the benefits that the medial buttress plate brings to the patient in terms of maintenance of fracture stability. Which should be noticed is that, similar to the results reported by Lucas ${ }^{14}, 2$ patients in the CBMP group had impingement with hip flexion, which is probably due to the plate being too proximal.

There are some limitations to our study. First, given that this is retrospective data from the same institution with a relatively small sample size, prudent attitude should be adopted regarding the interpretations of our outcomes. Second, in order to get more persuasive and generalizable results, more comprehensive studies should be conducted, such as multicenter trials with large sample sizes.

\section{Conclusions}

Anatomic reduction and stable fixation contribute to satisfactory outcomes in the treatment of nonelderly displaced femoral neck fractures. A buttress plate in the medial region of the femur neck with cannulated screws offers patients the best biomechanical microenvironment needed for fracture healing without obviously blood-supply disruption, especially in Pauwels type $₫$ and type III.

\section{Abbreviations}

MCS: multiple cannulated screws;

CMBP: combined with medial buttress plate;

CPM: continuous passive motion;

HHS: The Harris Hip Score;

EQ-5D: EuroQol five-dimension questionnaire;

SD: Standard deviation;

MFCA: The medial femoral circumflex artery;

SRA: superior retinacular artery;

IRA: inferior retinacular artery.

\section{Declarations}




\section{Ethics approval and consent to participate}

This retrospective study was approved by the Ethics Committee of The First Affiliated Hospital of Xinjiang Medical University. All methods were performed in accordance with the Declarations of Helsinki. Written informed consent was obtained from all patients for their data to be recorded in our study.

\section{Consent for publication}

Not applicable.

\section{Availability of data and materials}

The datasets analyzed during the current study are available from the corresponding author on reasonable request.

\section{Competing interests}

The authors declare that they have no competing interests.

\section{Funding}

This work was supported by National Natural Science Foundation of China grant number 81660368 .

\section{Authors' contributions}

CM and YSL contributes equally to this study.

CM, YSL: Conducted the study, collected, analyzed and interpreted the data, prepared the manuscript draft.

JLL: Collected and interpreted the data.

LC: Created and statistical analyzed the data.

$\mathrm{JYH}$ : Analyzed and interpreted the data.

XFL: Designed the study, reviewed the manuscript.

ZRX: Supervised the study, reviewed the manuscript.

Approval of the final draft: CM, YSL, JLL, LC, JYH, XFL, ZRX.

\section{Acknowledgements}

Not applicable.

\section{Author details}


${ }^{1}$ Department of Trauma Orthopedics, The First Affiliated Hospital of Xinjiang Medical University, Urumqi, China.

${ }^{2}$ Department of Microrepair and Reconstruction, The First Affiliated Hospital of Xinjiang Medical University, Urumqi, China.

${ }^{3}$ Department of Prosthodontics, The First Affiliated Hospital of Xinjiang Medical University, Urumqi, China.

${ }^{4}$ Department of Ophthalmology, The First Affiliated Hospital of Xinjiang Medical University, Urumqi, China.

\section{References}

1. Robinson $\mathrm{CM}$, Court-Brown $\mathrm{CM}, \mathrm{McQueen} \mathrm{MM}$, Christie J. Hip fractures in adults younger than 50 years of age. Epidemiology and results. Clin Orthop Relat Res 1995:238-46.

2. Swiontkowski MF. Intracapsular fractures of the hip. J BONE JOINT SURG AM 1994;76:129-38.

3. Mir $\mathrm{H}$, Collinge $\mathrm{C}$. Application of a medial buttress plate may prevent many treatment failures seen after fixation of vertical femoral neck fractures in young adults. MED HYPOTHESES 2015;84:42933.

4. Davidovitch RI, Jordan CJ, Egol KA, Vrahas MS. Challenges in the treatment of femoral neck fractures in the nonelderly adult. J Trauma 2010;68:236-42.

5. Liporace F, Gaines R, Collinge C, Haidukewych GJ. Results of internal fixation of Pauwels type-3 vertical femoral neck fractures. J BONE JOINT SURG AM 2008;90:1654-9.

6. Wang Z, Yin Y, Li Q, Sun G, Peng X, Yin H, et al. Comparison of early complications between the use of a cannulated screw locking plate and multiple cancellous screws in the treatment of displaced intracapsular hip fractures in young adults: a randomized controlled clinical trial. J ORTHOP SURG RES 2018;13:201.

7. Luttrell $\mathrm{K}$, Beltran $\mathrm{M}$, Collinge $\mathrm{CA}$. Preoperative decision making in the treatment of high-angle "vertical" femoral neck fractures in young adult patients. An expert opinion survey of the Orthopaedic Trauma Association's (OTA) membership. J ORTHOP TRAUMA 2014;28:e221-5.

8. Aminian A, Gao F, Fedoriw WW, Zhang LQ, Kalainov DM, Merk BR. Vertically oriented femoral neck fractures: mechanical analysis of four fixation techniques. J ORTHOP TRAUMA 2007;21:544-8.

9. Augat P, Bliven E, Hackl S. Biomechanics of Femoral Neck Fractures and Implications for Fixation. J ORTHOP TRAUMA 2019;33 Suppl 1:S27-32.

10. Siavashi B, Aalirezaei A, Moosavi M, Golbakhsh MR, Savadkoohi D, Zehtab MJ. A comparative study between multiple cannulated screws and dynamic hip screw for fixation of femoral neck fracture in adults. INT ORTHOP 2015;39:2069-71. 
11. Harris WH. Traumatic arthritis of the hip after dislocation and acetabular fractures: treatment by mold arthroplasty. An end-result study using a new method of result evaluation. J BONE JOINT SURG AM 1969;51:737-55.

12. Ye Y, Chen K, Tian K, Li W, Mauffrey C, Hak DJ. Medial buttress plate augmentation of cannulated screw fixation in vertically unstable femoral neck fractures: Surgical technique and preliminary results. INJURY 2017;48:2189-93.

13. Steinberg ME, Hayken GD, Steinberg DR. A quantitative system for staging avascular necrosis. J Bone Joint Surg Br 1995;77:34-41.

14. Marchand LS, Karns M, Higgins TF, Aoki SK. Femoral Neck Fracture Fixation with a Medial Buttress Plate That Led to Impingement with Hip Flexion: A Case Report. JBJS Case Connect 2019;9:e21.

15. Nwankwo CD, Schimoler P, Greco V, Kharlamov A, Westrick ER, Miller MC. Medial Plating of Pauwels Type III Femoral Neck Fractures Decreases Shear and Angular Displacement Compared to Derotational Screw. J ORTHOP TRAUMA 2020.

16. Kunapuli SC, Schramski MJ, Lee AS, Popovich JJ, Cholewicki J, Reeves NP, et al. Biomechanical analysis of augmented plate fixation for the treatment of vertical shear femoral neck fractures. $J$ ORTHOP TRAUMA 2015;29:144-50.

17. Giordano V, Alves DD, Paes RP, Amaral AB, Giordano M, Belangero W, et al. The role of the medial plate for Pauwels type III femoral neck fracture: a comparative mechanical study using two fixations with cannulated screws. J Exp Orthop 2019;6:18.

18. Della RG. Gaps and opportunities in the management of the young femoral neck fracture. INJURY 2015;46:515-8.

19. Jo S, Lee SH, Lee HJ. The Correlation between the Fracture Types and the Complications after Internal Fixation of the Femoral Neck Fractures. Hip Pelvis 2016;28:35-42.

20. Zhang YL, Chen S, Ai ZS, Gao YS, Mei J, Zhang CQ. Osteonecrosis of the femoral head, nonunion and potential risk factors in Pauwels grade- 3 femoral neck fractures: A retrospective cohort study. Medicine (Baltimore) 2016;95:e3706.

21. Boraiah S, Dyke JP, Hettrich C, Parker RJ, Miller A, Helfet D, et al. Assessment of vascularity of the femoral head using gadolinium (Gd-DTPA)-enhanced magnetic resonance imaging: a cadaver study. J Bone Joint Surg Br 2009;91:131-7.

22. TRUETA J, HARRISON MH. The normal vascular anatomy of the femoral head in adult man. J Bone Joint Surg Br 1953;35-B:442-61.

23. Putnam SM, Collinge CA, Gardner MJ, Ricci WM, McAndrew CM. Vascular Anatomy of the Medial Femoral Neck and Implications for Surface Plate Fixation. J ORTHOP TRAUMA 2019;33:111-5.

24. Kalhor M, Horowitz K, Gharehdaghi J, Beck M, Ganz R. Anatomic variations in femoral head circulation. HIP INT 2012;22:307-12.

25. Lazaro LE, Sculco PK, Pardee NC, Klinger CE, Dyke JP, Helfet DL, et al. Assessment of femoral head and head-neck junction perfusion following surgical hip dislocation using gadolinium-enhanced magnetic resonance imaging: a cadaveric study. J BONE JOINT SURG AM 2013;95:e1821-8. 
26. Swiontkowski MF, Winquist RA, Hansen SJ. Fractures of the femoral neck in patients between the ages of twelve and forty-nine years. J BONE JOINT SURG AM 1984;66:837-46.

\section{Figures}
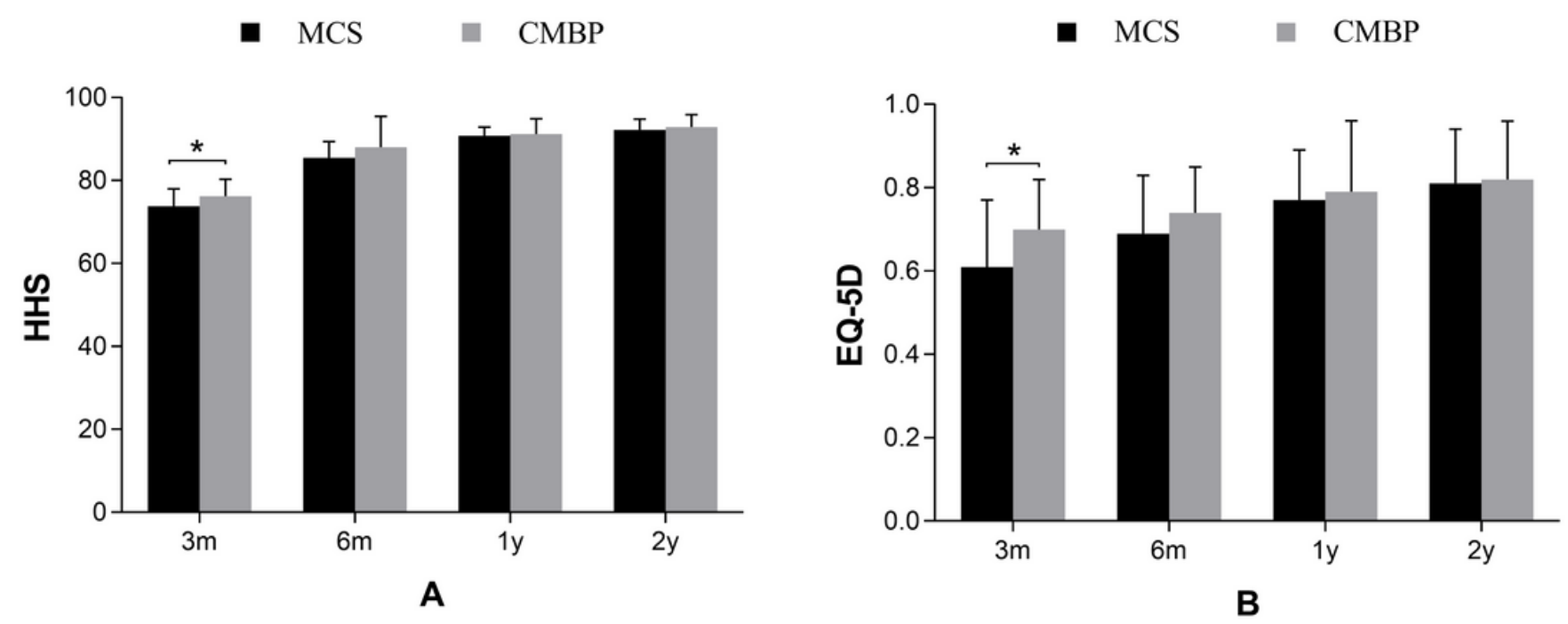

Figure 1

The HHS and EQ-5D of MCS and CMBP group (Pauwels type $\triangle$ and $\triangle$ ). MCS: multiple cannulated screws group. CMBP: combined with medial buttress plate group. * significant difference between two groups $(p<0.05)$. 